\title{
USING VSWIR MICROIMAGING SPECTROSCOPY TO EXPLORE THE MINERALOGICAL DIVERSITY OF HED METEORITES
}

\author{
Abigail A. Fraeman ${ }^{1}$, Bethany L. Ehlmann ${ }^{1,2}$, Geraint W.D. Northwood-Smith ${ }^{2,3}$, Yang Liu ${ }^{1}$, Meenakshi \\ Wadhwa ${ }^{4}$, Rebecca N. Greenberger ${ }^{1}$ \\ ${ }^{1}$ Jet Propulsion Laboratory, California Institute of Technology ${ }^{2}$ California Institute of Technology \\ ${ }^{3}$ Cambridge University ${ }^{4}$ Arizona State University
}

\begin{abstract}
We use VSWIR microimaging spectroscopy to survey the spectral diversity of HED meteorites at $80-\mu \mathrm{m} /$ pixel spatial scale. Our goal in this work is both to explore the emerging capabilities of microimaging VSWIR spectroscopy and to contribute to understanding the petrologic diversity of the HED suite and the evolution of Vesta. Using a combination of manual and automated hyperspectral classification techniques, we identify four major classes of materials based on VSWIR absorptions that include pyroxene, olivine, Febearing feldspars, and glass-bearing/featureless materials. Results show microimaging spectroscopy is an effective method for rapidly and non-destructively characterizing small compositional variations of meteorite samples and for locating rare phases for possible follow-up investigation. Future work will include incorporating SEM/EDS results to quantify sources of spectral variability and placing observations within a broader geologic framework of the differentiation and evolution of Vesta.
\end{abstract}

Index Terms - Microimaging spectroscopy, VSWIR spectroscopy, HED meteorites, Vesta, spectral classification

\section{INTRODUCTION}

Visible-short wavelength infrared (VSWIR) imaging spectroscopy at spatial resolutions from the meter to micrometer spatial scale has recently emerged as a powerful technique for rapidly mapping the composition of geologic samples [1]. This non-destructive method provides information about mineralogical and elemental variability while maintaining textural context, allowing for new insights into geological processes. Mapping the spectral properties of laboratory analog samples at multiple spatial scales also allows for a better understanding of how small-scale compositional heterogeneities may influence meter to kilometer scale space-based VSWIR spectral remote sensing observations of planetary bodies $[1,2]$. VSWIR microimaging spectrometers have also been proposed for in situ science on planetary bodies as a means to rapidly assess petrology (composition and texture) at sub-mm scales with little to no sample preparation $[3,4]$.

Here we use VSWIR microimaging spectroscopy, i.e., imaging spectroscopy at micrometers spatial scale, to survey the spectral diversity of the howardite, eucrite, and diogenite (HED) meteorite suite at $80-\mu \mathrm{m} / \mathrm{pixel}$. The petrology and spectral properties of HED meteorite powders and bulk chips have been studied extensively, and their resemblance to telescopic spectra of the asteroid 4 Vesta led to the conclusion that this asteroid was a strong candidate to be the HED parent body [e.g. 5, 6]. Subsequently, the Visible and Infrared Imaging Spectrometer (VIR) on the Dawn spacecraft mapped the spectral properties of the surface of 4 Vesta from 0.4 to 5 micrometers up to $43 \mathrm{~m} /$ pixel [7]. Both spectra from Vesta and the HED meteorites are dominated by broad absorptions near 1 and $2 \mu \mathrm{m}$, consistent with the mineral pyroxene. Variations in the shapes and local minima of these two absorption features indicate variations in the pyroxene's $\mathrm{Ca}, \mathrm{Fe}$, and $\mathrm{Mg}$ content, which are indicators of cooling and crystallization history. Eucrites are comprised primarily of low-Ca pyroxene and plagioclase feldspar and are thought to have formed near or on the surface of Vesta. Diogenites are mineralogically diverse but are primarily magnesian orthopyroxene and are thought to have likely cooled deeper within Vesta's crust. Howardites are regolith breccias comprised of a mixture of both eucrite and diogenite fragments [8].

Our goal in this work is to use the capabilities of microimaging VSWIR spectroscopy to contribute to understanding the petrologic diversity of the HED suite and the evolution of Vesta by (1) resolving spectral end members - i.e., spectra of the mineral constituents of Vesta - for use in interpretation of infrared remote sensing data from the Dawn spacecraft, (2) locating rare phases that can be examined using other detailed analytical techniques, and (3) non-destructively and rapidly classifying large numbers of meteorites, including estimating their modal mineralogy within a petrographic context. Here we focus on methods for resolving spectral endmembers and finding rare phases and demonstrate the effectiveness of microimaging spectroscopy for rapidly characterizing geologic samples. 

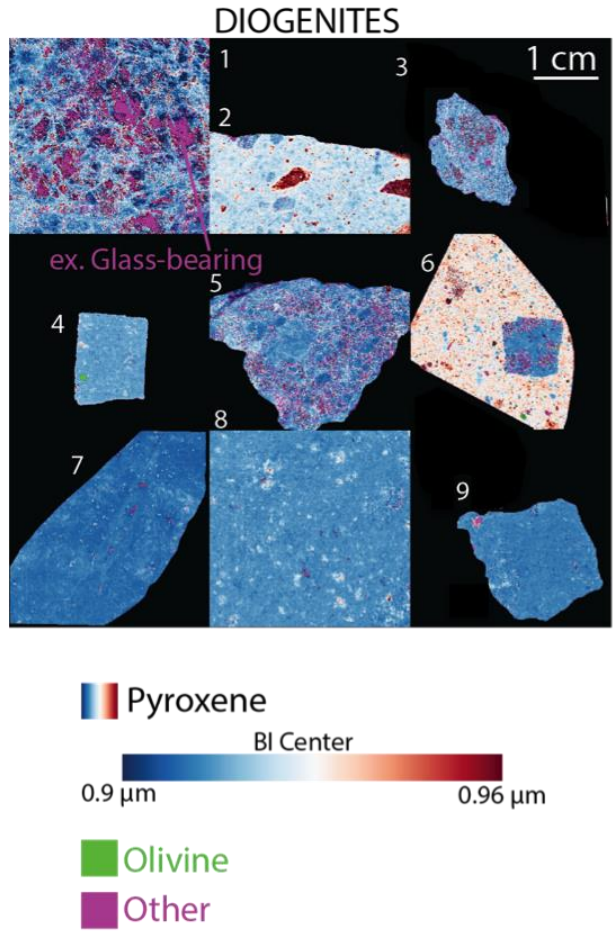
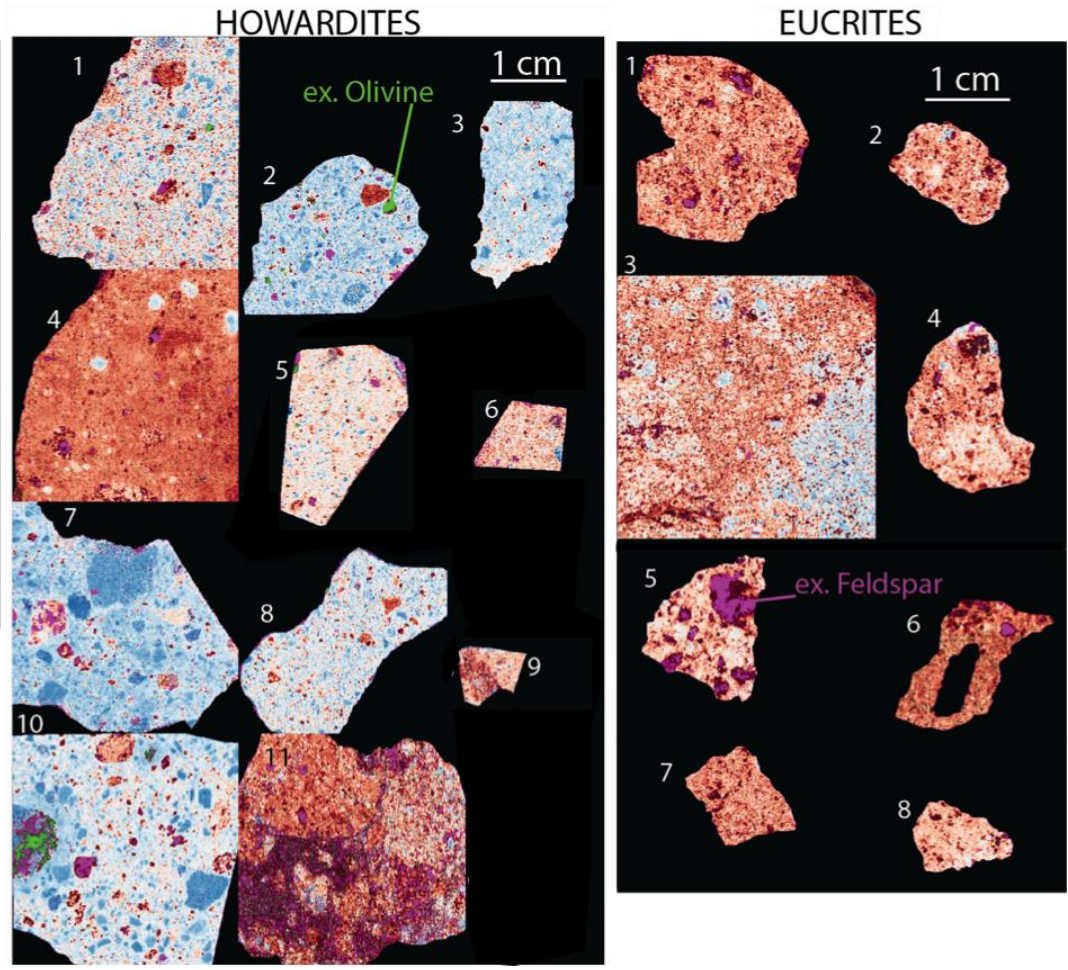

Figure 1: False color images of HEDs, acquired with UCIS, showing spectral diversity in igneous minerals and glasses. Pyroxene, defined as having bands near $1 \mu \mathrm{m}$ and $2 \mu \mathrm{m}$ with depths $>5 \%$, is shown in blue and red. Pyroxenes with $\mathrm{BI}$ centers near $0.9 \mu \mathrm{m}$ are blue and band centers near $0.96 \mu \mathrm{m}$ are red). Olivine grains, defined as having OLINDEX [8] $>0.3$ are shown in green, and other classes, including feldspar- and glass-bearing, are purple. "Ex" points to example grains identified as olivine, feldspar, and glass-bearing. Sample IDs: Diogenites -- (1) Northwest Africa 7831 (2) Los Vientos 001 (3) Northwest Africa 2629 (4) Aioun el Atrouss (5) Northwest Africa 6074 (6) Northwest Africa 1648 (7) Bilanga (8) Johnstown (10) Shalka. Howardites - (1) Old Homestead 001 (2) Northwest Africa 7127 (3) Northwest Africa 2060 (4) Northwest Africa 1929 (5) Northwest Africa 1769 (6) Northwest Africa 1769 (7) Frankfort (stone) (8) Kapoeta (9) Northwest Africa 1282 (10) Northwest Africa 6920 (11) Muckera 002. Eucrites - (1) Stannern (2) Sioux County (3) Northwest Africa 1908 (4) Sioux County (5) Juvinas (6) Pasamonte (7) Haraiya (8) Haraiya.

\section{METHODS}

We acquired 11 howardite, 8 eucrite, and 9 diogenite meteorite fragments from the ASU Center for Meteorite Studies, the Caltech mineral collection, Y. Liu, and G. Rossman, and these samples were analyzed using JPL's Ultra-Compact Imaging Spectrometer (UCIS) (Fig. 1). UCIS is a small imaging spectrometer prototype designed to be incorporated on future rover or lander interplanetary missions [2]. Through the use of different front optics, the spectrometer can operate in either a telescopic or microscopic imaging mode suitable for analyzing meterscale outcrops in the field or centimeter-scale laboratory samples respectively. In the microimaging mode utilized in this study, UCIS collects spectra from samples at 81 $\mu \mathrm{m} /$ pixel across 213 discrete wavelengths from $0.5-2.5$ $\mu \mathrm{m}$. The spectral resolution is approximately $10 \mathrm{~nm}$. A single scan over a $\sim 3 \mathrm{~cm} \times 3 \mathrm{~cm}$ area takes $\sim 3-5$ minutes to complete, depending on exposure settings.
An initial assessment of sample spectral diversity was performed using a combination of manual investigation and a variety of near-automated techniques available in the ENVI software package [9]. Spectral image cubes were first processed using a minimum-noise fraction (MNF) transformation to reduce random spectral noise. Next, the Ndimensional visualization tool was used to locate spectral endmembers within a scene, and spectral angle mapping (SAM) was used to classify an image using the given endmembers. SAM results were interpreted to address the adequacy of the hand selected endmembers in fully characterizing the scene, and more endmembers were selected using $\mathrm{N}$-dimensional visualization if deemed necessary. Spectral parameter mapping designed to search for the presence of absorption features in locations of known geologic materials was also employed [10]. 


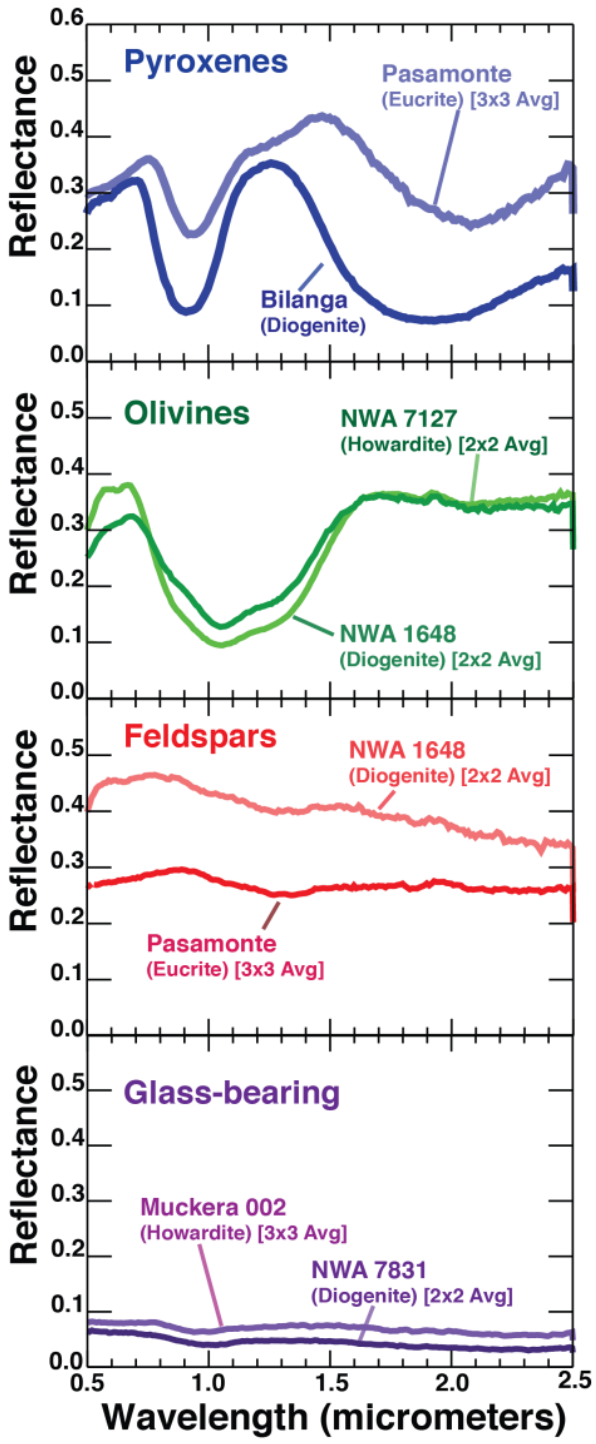

Figure 2: Example spectra representative of each of the four major spectral types observed. Source samples for each spectrum are labeled in the left, as well as number of pixels averaged together if applicable.

\section{SPECTRAL DIVERSITY}

We identify four major classes of materials based on VSWIR absorptions. These spectral classes are consistent with pyroxenes, olivines, Fe-bearing feldspars, and glassbearing/featureless materials (Fig. 2). The relative prevalence of each endmember can be seen in Fig. 1. There is some spectral diversity within each class that is partially indicative of elemental substitutions. The validity of major class identifications were confirmed in select spots in samples NWA 1769 (both fragments), and NWA 6920 using SEM/EDS point chemical analyses.

\subsection{Pyroxene}

Pyroxene is characterized by a pair of broad absorptions centered near 1 and $2 \mu \mathrm{m}$ caused by $\mathrm{Fe}^{2+}$ in the M1 and M2 sites of the pyroxene crystal structure and referred to as band 1 (B1) and band 2 (BII) (Fig. 2). Pyroxene was the most prevalent spectral class for all of the investigated meteorite samples, consistent with infrared spectra of bulk powdered HED spectra that resemble pyroxene [e.g.,11] and km-scale spectra from Dawn at Vesta [e.g., 7].

The positions of BI and BII absorption centers, which are controlled primarily by $\mathrm{Fe}$ - and $\mathrm{Ca}$-content in pyroxene, were calculated using the method of [12], modified to fit the bands using a $6^{\text {th }}$ order polynomial. On the whole, band centers are consistent with previous measurements of bulk HED spectra [11, 12], although there are some intriguing trends that become apparent only at the small spatial resolution (Fig. 3). Single pixel pyroxene spectra from eurcrites and howardites plot mostly within the two fields established in prior studies, but the absorption positions differ for diogenites, and there appear to be two spectral classes within the diogenite field.

\subsection{Olivine}

Olivine is a rare phase in howardites and diogenites [6], but we have been able to easily identify a handful of olivine grains in several of our samples. Olivine spectra are characterized by broad bands near $1 \mu \mathrm{m}$ and $1.3 \mu \mathrm{m}$ (Fig. 2), and in many cases appear to be mixed with pyroxene in the HEDs at the scale of UCIS measurement, based on the presence of a weak $2 \mu \mathrm{m}$ feature. A few grains are large enough, however, that they appear spectrally relatively pure.

\subsection{Feldspar}

Feldspar is typically bright and spectrally featureless in the VSIWR wavelength range, although it sometimes has a weak absorption near $1.3 \mu \mathrm{m}$ due to Fe-substitution; this is observed in the HED samples. Although feldspar has not been observed directly in Dawn and HED spectral data, it is known to be a major phase from HED meteorite studies [8] and is undoubtedly an important component driving Vesta's bulk spectral properties.

\subsection{Low albedo, relatively featureless materials}

This class is defined by its low overall albedo and weak to non-existent absorption features. This class represents glassbearing materials, impact melts, or opaque phases such as troilite and/or carbonaceous materials. Select samples 

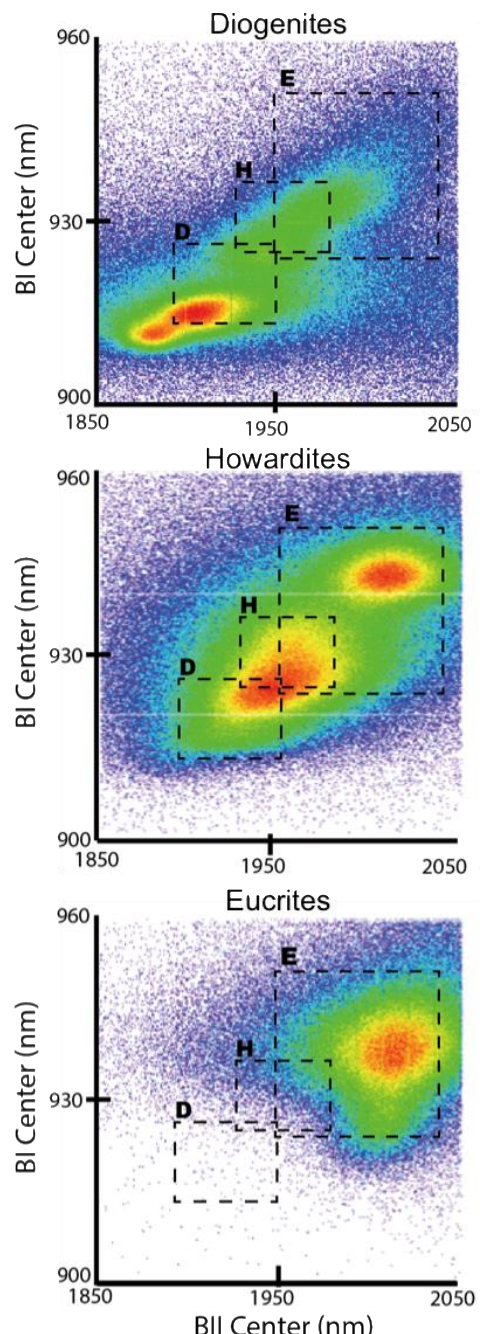

Figure 3: Pyroxene band I and band II center variability within HED samples. Average values for band centers from bulk HED meteorites compiled in [5] fall within dotted boxes. Data are density sliced such that red is high density and purple is low.

identified as glasses have weak absorptions near $1.0 \mu \mathrm{m}$ and $2.0 \mu \mathrm{m}$, consistent with the composition and spectral properties of mafic glasses.

\section{CONCLUSIONS AND FUTURE WORK}

Microimaging spectroscopy is an effective method for rapidly and non-destructively characterizing compositional variations in meteorite samples. The sensitivity of this method to compositional differences is evidenced by the significant spectral diversity within the pyroxene class spectra both between howardites, eucrites, and diogenites and within individual meteorite samples (Fig. 1, 3). Future work with spatially coregistered SEM/EDS will determine whether these differences are due to compositional differences, the effects of impact shock, or sub-pixel mixtures of multiple phases.

Microimaging spectroscopy is also successfully used to locate uncommon, scientifically important phases that may be investigated in follow-up studies. For example, highlighting the abundance and locations of olivine grains for followup study with other high resolution techniques (e.g., trace element analyses with microprobe) may provide important clues towards understanding the differentiation of Vesta. The magma ocean model for Vesta's differentiation predicts that olivine will have been preferentially concentrated deep within the mantle and therefore should be exposed in Vesta's deepest impact craters [13]. One of the surprising results of the Dawn mission was the lack of olivine spectral signatures associated with deep basins and presence of olivine spectral signatures at unexpected locations [14]. One hypothesis to explain the lack of olivine spectral signatures in the deepest basins is that the mineral is not present in great enough quantities to be detectable when mixed with pyroxene, and some have argued that the olivine spectral signatures that were detected on Vesta are representative of exogenous material rather and indigenous Vestan mantle material [15]. Because of their importance in testing predictions of Vesta's differentiation, the olivine grains identified using the UCIS data may be targets for future studies designed to understand their origin [16] and/or to test radiative transfer models for Vesta's surface which predict up to 10-20 weight \% olivine could be present on Vesta but masked due to mixing with spectrally dominant pyroxene $[17,18]$.

Future work will include incorporating SEM/EDS results to quantify sources of spectral variability at the micrometer scale and placing observations within a broader geologic framework of the differentiation and evolution of Vesta.

Acknowledgements-The research was partially carried out at the Jet Propulsion Laboratory, California Institute of Technology, under a contract with the National Aeronautics and Space Administration. (c) 2016. All rights reserved. Thanks to a W.M Keck Institute for Space Studies Postdoctoral Fellowship, Caltech GPS Division Texaco Postdoctoral Fellow, and JPL RTD grant to A.A.F., and a JPL PDF/RTD program grant to B.L.E. and D. Blaney for partial support of this work.

\section{REFERENCES}

[1] R. N. Greenberger, J. F. Mustard, B. L. Ehlmann, D. L. Blaney, E. A. Cloutis, J. H. Wilson, R. O. Green, and A. A. Fraeman, 
"Imaging spectroscopy of geological samples and outcrops: Novel insights from microns to meters", GSA Today, pp. 4-10, 2015.

[2] B. Van Gorp, P. Mouroulis, D.L. Blaney, R.O. Green, B. L. Ehlmann, and J. I. Rodriguez, "Ultra-compact imaging spectrometer for remote, in situ, and microscopic planetary mineralogy," J. Appl. Remote Sens. 8(1), 084988, 2014.

[3] B.L., Ehlmann, P. Mouroulis, B. Van Gorp, R. Green, D. Blaney, J. Rodriguez, J. Mustard, S. Murchie, C. Herd, F. Seelos, and S. Feldman, "Microimaging VSWIR Spectroscopy Instruments for Planetary Exploration: Measuring In-Situ Mineralogy, Ices, Organics, and Linking to Remote Observation," International Workshop on Instrumentation for Planetary Missions, abs. 1046.

[4] R.O. Green, B.L. Ehlmann, A.A. Fraeman, D. Blaney, Y. Liu, N. Chabot, S.L. Murchie, M. Wadhwa, C.D.K. Herd, M.A. Velbel, P. Mourolis, B. Van Gorp, "Microimaging spectroscopy for the exploration of small bodies: first laboratory measurements of carbonaceous chondrite and HED meteoerites and a proposed M6 instrument for in situ measurement," Lunar \& Plan Sci. Conf., abstract \#2154, 2015.

[5] T.B. McCord, J.B. Adams, and T.V. Johnson. "Asteroid Vesta: Spectral Reflectivity and Compositional Implications," Science, 168, 1445-1447. 1970.

[6] G. J. Consolmagno and M.J. Drake. "Composition and evolution of the eucrite parent body; evidence from rare earth elements," Geochim. Cosmochim. Acta, 41, 1271-1282. 1977.

[7] M. C. De Sanctis, E. Ammannito, M. T. Capria, F. Tosi, F. Capaccioni, F. Zambon, F. Carraro, S. Fonte, A Frigeri, R. Jaumann, G. Magni, S. Marchi, T. B. McCord, L.A. McFadden, H. Y. McSween, D. W. Mittlefehldt, A. Nathues, E. Palomba, C. M. Pieters, C. A. Raymond, C. T. Russell, M. J. Toplis, and D. Turrini, "Spectroscopic Characterization of Mineralogy and Its Diversity Across Vesta," Science, 336, 6082, 697-700. 2012.

[8] D. W. Mittlefehldt, "Asteroid (4) Vesta: I. The howarditeeucrite-diogenite (HED) clan of meteorites," Chemie der Erde, 75, 155-185. 2015.

\section{[9] Exelis Visual Information Solutions (2010)}

[10] C. E. Viviano-Beck, F. P. Seelos, S. L. Murchie, E. G. Kahn, K. D. Seelos, H. W. Taylor, K. Taylor, B. L. Ehlmann, S. M. Wisemann, J. F. Mustard, and M. F. Morgan, "Revised CRISM spectral parameters and summary products based on the currently detected mineral diversity on Mars," Journal of Geophysical Research: Planets, 119, 1403-1431. 2014.

[11] E. A. Cloutis, M. R. M. Izawa, L. Pompilio, V. Reddy, H. Hiesinger, A. Nathues, P. Mann, L. Le Corre, E. Palomba, and J.F. Bell III, "Spectral reflectance properties of HED meteorites + CM2 carbonaceous chondrites: Comparison of HED grain size and compositional variations and implications for the nature of low- albedo features on Asteroid 4 Vesta," Icarus, 223, 2, 850-877. 2013.

[12] E. Ammannito, M. C. De Sanctis, F. Capaccioni, M T. Capria, F. Carraro, J-P. Combe, S. Fonte, A. Frigeri, S. P. Joy, A. Longobardo, G. Magni, S. Marchi, T. B. McCord, L. A. McFadden, H. Y. McSween, E. Palomba, C. M. Pieters, C. A. Polanskey, C. A. Raymond, J. M. Sunshine, F. Tosi, F. Zambon, and C. T. Russell, "Vestan lithologies mapped by the visual and infrared spectrometer on Dawn," Meteoritics \& Planetary Science, 48, 11, 2185-2198. 2013.

[13] H. Y. McSween, E. Ammannito, V. Reddy, T. H. Prettyman, A. W. Beck, M. C. De Sanctis, A. Nathues, L. Le Corre, D. P. O'Brien, N. Yamashita, T. J. McCoy, D. W. Mittlefehldt, M. J. Toplis, P. Schenk, E. Palomba, D. Turrini, F. Tosi, F. Zambon, A. Longobardo, F. Capaccioni, C. A. Raymond, and C. T. Russell, "Composition of the Rheasilvia basin, a window into Vesta's interior," Journal of Geophysical Research: Planets, 118, 2, 335 346. 2013.

[14] E. Ammannito, M. C. De Sanctis, E. Palomba, A. Longobardo, D. W. Mittlefehldt, H. Y. McSween, S. Marchi, M. T. Capria, F. Capaccioni, A. Frigeri, C. M. Pieters, O. Ruesch, F. Tosi, F. Zambon, F. Carraro, S. Fonte, H. Hiesinger, G. Magni, L. A. McFadden, C. A. Raymond, C. T. Russell and J. M. Sunshine, "Olivine in an unexpected location on Vesta's surface," Nature, 504, 122-125. 2013.

[15] L. Le Corre, V. Reddy, J. A. Sanchez, T. Dunn, E. A. Clouis, M. R. M. Izawa, P. Mann, and A. Nathues. "Exploring exogenic sources of the olivine on Asteroid (4) Vesta," Icarus, 258, 483499. 2015.

[16] N. G. Lunning, H. Y. McSween, T. J. Tenner, N. T. Kita, R. J. Bodnar, "Olivine and pyroxene from the mantle of asteroid 4 Vesta," Earth and Planetary Science Letters, 418, 126-135. 2015.

[17] F. Poulet, O. Ruesch, Y. Langevin, H. Hiesinger, "Modal mineralogy of the surface of Vesta: Evidence for ubiquitous olivine and identification of meteorite analogue," Icarus, 253, 364-377. 2015.

[18] S. Li and R. Milliken, "Estimating the modal mineralogy of eucrite and diogenite meteorites using visible-near infrared reflectance spectroscopy," Meteoritics \& Planetary Science, 50, 11, 1821-1850. 2015. 